\title{
Migrations and Intergenerational Religious Transmission: Issues from International Literature
}

\author{
Donatella Bramanti, Stefania Meda and Giovanna Rossi
}

\section{1 Introduction}

The aim of this chapter is to provide an overview of international literature about the importance of religion in family migration histories, with a special focus on how religious values are transmitted between generations.

The significant presence of migrant families having a clear religious identity, which they keep nurturing in their host country (USA, Canada, France and the Netherlands), for at least the last thirty years has brought about the need to explore the issue of religion, in order to understand its significance within the processes aimed at integrating migrant minorities. Today, we are facing one further issue, which has been investigated by research less extensively and which is the focus of this analysis, concerning the fact that the reason behind migration is a specific religious affiliation.

Giving consideration to the connection between religion and migrant families seems quite arduous in our highly secularized society, where the topic of religion appears to be in the background and is often regarded as playing a barely active role in guiding choices and strategies to face the challenges and risks related to families' and individuals' migration.

The following critical issues tend to overlap in this topic:

a) The phenomenon of religious persecution of Christian minorities in their home countries, which affects several families migrating to Europe and to Italy in particular, shifts the focus back onto the debate about religious belonging, which has gradually been playing a secondary role with the advent of the secularization process. This process is now apparently suffering a setback, and religious belonging is once again playing a primary role (Rizzi, 2016; see also Chapter 3 ).

b) On the other hand, the secularization process (Eberstadt, 2013) -which has been present, at least so far, to some extent in Western Europe, as well as in the English-speaking world, since the second half of the 2oth century- has been documented by several studies in Europe (Pollini et al., 
2012). These studies underline how religiousness has suffered a steady decline in its role as a pillar of the transmission of values, in general, and also of the importance of the family.

c) But what is the outcome of secularization? The advent of a post-secular society, which is the outcome of the morphogenetic process of the previous secularized society. This process implies "a further differentiation between the essential values (let us say religious, for the sake of simplicity) that inspire the variety of cultures appearing in it" (Belardinelli et al., 2009: 110). In the Western world, where the phenomenon of migration is increasingly important, a process of differentiation from a cultural and religious point of view has been taking place over the last few decades. Today, the post-secular society is expressed by a multi-cultural society (Donati, 2008).

d) The post-secular society includes elements of paradoxicality as it incorporates both the unexpected effects of secularization (lack of meaning, deviant behaviors that can be classified as discomforts of modernity) and direct effects (need for transcendent meanings in irrational forms or forms of new rationality) (Donati, 2009). As for Europeans, on the one hand there is a progressive decrease in the importance of religious belonging, and, on the other hand, there is a sort of re-sacralization of social bonds (sometimes required by non-Christian religions) and of new forms of religious participation.

e) The behavior of migrant families having a clear religious identity can be explained in the same perspective of paradoxicality. Two different processes emerge: a sort of theologizing experience (Connor, 2009; Massey, Higgins, 2011) -which is a sign of a higher level of personal awareness, but will not always affect the communal religious participation-, and the disaffiliation from religious practices and beliefs.

f) It is also necessary to avoid any generalization when referring to the word generation. What do we mean by it today? Generation is a group of people sharing one relationship (Boccacin, 2005), that is the one linking their position within one's progeny in the family-relatives sphere (i.e. child, parent, grandparent, etc.) with the well-defined position in the societal sphere depending on the social age (i.e. depending on the relevant age group: young, adult, elderly, etc.) (Attias-Donfut, 2000; Rossi, 2012).

g) Lastly, what do we mean by religion? Religion differs from culture (rituals, myths, sacred, etc.) when "it becomes the relationship with the entirely other than me (transcendence) that is within me and outside of me at the same time" (Donati, 2009: 304). However, differences between 
religions can be observed by considering the idea of transcendence. This produces social effects that can be very diverse; in particular, "the difference of Christianity lies in referring to a theological matrix that uses a relational semantics of difference" (Donati, 2010: 218) thus not limiting religion to a series of practices and/or ritual obligations (Pace, 2013).

\section{2 \\ Intergenerational Transmission of Religious Values: the Main Issues Emerging from Italian and International Studies}

The transmission of values falls within the realm of socialization processes and -from a relational sociology perspective- can be seen as a process aimed at "transmitting the ethos of a culture, which expresses a normative, i.e. interpretive, view on life and on the world that individuals have despite not being their own only makers" (Donati, 1989: 98). Values and norms are part of a story, a memory, and are constantly comprehended, endorsed and modified within relationships, thus eventually becoming an interpretive project (Donati, 1991). Socialization is virtuously achieved when shifting from a unidirectional model to a transactional model implying reciprocity between individuals (in this case, adults-grandparents, parents-children, grandparents-grandchildren).

The intergenerational transmission of values, which plays a crucial role in the socialization process (Pontalti, Rossi, 1995; Cigoli, 1995; Barni, 2009), includes two logs, the first one recording what lies between generations and which can be defined as intergenerational transmission, and the second one concerning what goes beyond, what crosses and transcends generations, by exceeding them - which has been defined as transgenerational transmission in psychosocial literature (Cigoli, Scabini, 2006). The intergenerational transmission of values refers to the system of moral principles that previous generations pass down to the following ones (whether they pass it down and how) and to what the latter, in turn, perceive, accept, or refuse to pass down (Scabini, 2006). The transmission of religion has specific features that have long been underestimated and appear significant in relationships between generations.

One of the issues that several studies on migration, families and religion focus on is linked to the question whether religion is a bridge or a barrier to the inclusion of families (Foner, Alba, 2008). The findings of the study conducted on Coptic families and their children in the following chapters provide interesting elements on this matter.

The act of thinking by generations involves a significant heuristic ability: this approach actually allows to observe relationships between generations and 
the symbolic content that is generated through them and is transmitted within families and society. Thinking by generations implies bringing into focus a different, mutual responsibility: previous generations actually play a crucial role in favoring or hindering the chances that the following generations could be or not be generative. In our opinion, the meaning of the whole generation concept lies in the bond, in the significance that such bond assumes on a personal level for those who engage in it in everyday life, and in the significance, within a social context, that the intergenerational bond, engaged both inside and outside the family, assumes in the surrounding context (meso level) as well as in a wider, or social, context (macro level). In this perspective, the concept of generation is based, on the one hand, on the historical-biological age, in terms of descendant/ancestor relationships (family axis), and, on the other hand, on the mediation that society, and welfare systems in particular, provides to these bonds (social axis).

The concept of generation, thus, appears as a synthesis between the family and social spheres, and allows to place generations within the complex dynamics of resource and general exchange allocation.

Through the relational approach, it is possible to go beyond the previously quoted and predominantly monosemic interpretations of the concept of generation, which, despite helping to clarify the various meanings of the word, prevent us from reaching a comprehensive and multi-dimensional understanding of it.

Focusing on relationships between generations also implies considering their ambivalence, which is generated by the considerable variety and fragmentary nature of the elements at stake in relationships, and is related to their elements of risk.

One way to deal with this ambivalence in family relationships between generations is favoring elements of obligation/subjection and keeping the marked cultural differences between generations in the background. The emancipation strategy stands at the other end of the spectrum; within this strategy, balance in family life and possible innovations are based on the convergence of all family members' cultural patterns and values (Lüscher, 2011).

The elements of risk in intergenerational relationships do not seem to be considered in most postmodern analyses and reflections. However, we know that relationships between generations are not immune from ambivalence and can therefore be either generative or degenerative. Transmission?

Let us now explore the main issues emerging from the most recent studies on the transmission of values in immigrant families, with a special focus on 
religious values, which have been carried out in Italy and other European and non-European countries.

The relationship between religion and family has been extensively studied by sociologists, who have provided religion with a role of both a dependent and an independent variable in socialization processes within families.

The following are the areas explored by researchers who have embraced the first hypothesis (religion as a dependent variable):

a) the intentional religious education provided by parents;

b) the family atmosphere, i.e. relationships based on trust and mutual respect;

c) the family structure, with reference to the presence of the marital dyad;

d) the group of peers, i.e. the community.

A second line of research identifies religion as an independent variable. In this case, the main focus for researchers is to determine whether and when religion is a crucial factor in how families work.

Notably, researchers have analyzed the impact of parents' religiousness on the strength of family bonds, especially couple relationships; religious practices as a strengthening element in the educational project; and the parents' religiousness as a strong predictor of juvenile religiousness.

Both these perspectives provide very interesting clues to understanding the dynamics of the transmission of values between generations and allow to develop a complex picture. Empirical Evidence

The main empirical evidence emerging from research can be regarded as a reflection of two macro topics: the relationship between family and society and the dynamics within generations.

As for the relationship between family and society, a key issue is the place that religion holds in the transmission of a sense of ethnic belonging and identity (Chen, 2002; Min, Kim, 2002; Rumbaut, 2006). It is actually one of the most important indicators of group identity (Verkuyten et al., 2012). Moreover, religions provide certainty of belonging, but also the support for many beliefs and lifestyles, such as intergenerational obligations, or family rituals. One key issue concerns the contribution that religious values can offer in the transmission of the most salient features of a cultural heritage (Warner, 2000). In intergenerational relationships, the issue of cultural reproduction arises when children start going to school. 


\subsection{The Relationship between Family and Society}

In general, the connection between family, cultural identities and religious institutions is a crucial element in the processes through which migrants seek to forge a system of meanings and to find a way of life for themselves and their children. Scholars (Ambrosini, 2007) have noted that, for Catholic families during the first migration flows to North America, aggregation around religious institutions was a way to preserve their languages and cultural traditions, while trying to adapt to an obscure and often hostile environment.

Catholic schools often represented an ideal place to help children avoid discrimination. Besides, Churches represented more than just a place of worship: they were places where people could meet, socialize, and form associations or informal groups. These religious environments also provided the chance to take on leadership roles and experience civic participation, which otherwise would not have been accessible in the surrounding society. To quote a classic text on this matter: "Church was the first line of defense behind which immigrants could preserve their group identity" (Warner, Srole, 1945: 160). In other words, for years Churches and religious associations have represented a clearinghouse that allowed immigrants to adapt to the new social context without losing their roots and social networks (support). This role has proven long lasting and capable of passing from one generation to another. Nowadays, this applies to a certain number of communities, not only to Catholics.

Sometimes, these forms of belonging lead individuals and families to strengthen their religious sentiment. The title of a study about religion and ethnicity among Indian immigrants in the United States (Kurien, 2002) is: We are better Hindus here. And actually most of the people who were interviewed reported that they had become more religious after arriving in the new country, as they started to ponder the meaning of their religious identity (see also Chapter 12), which they gave for granted in their home country, for the first time. Besides, while the traditional Hindu religious activity is not generally practiced in groups except for some celebrations, religious gatherings provide community support in a context of migration.

\subsection{Social Integration}

According to several scholars (Hirschman, 2004; Reitz et al., 2009), religion can act as a vehicle for a good level of integration into society, despite the fact that, after what happened on $9 / 11 / 2001$, Islam is regarded as a barrier to the integration process, especially in Europe (Foner, Alba, 2008), because the values it fosters are considered in stark contrast with the values fostered by the society of the host country. 
The widespread phenomenon of conversion to Christian Churches among Asian immigrants in the United States is a sign of the importance of faith for families that are trying to redefine their identities in a new environment.

It is more difficult to detect this kind of experience in a secularized context like the European one, in which, conversely, the loss of religious practices by migrants (and especially by second generations) is seen as a positive event, an indication of a full and successful assimilation (Tribalat, 1995).

Religious belonging appears to be a rather important factor according to the chances of social integration and to the cultural differences with the host country (see also Chapter 13).

Foner and Alba (2008) addressed the issue of differences in the importance of religion in Western countries. The assumption is that, in the United States, studies tend to focus more on religion as a factor in integration, whereas in Europe religion (and Islam in particular) is regarded as a barrier to immigrants' integration. These polarized perspectives reflect the different social dynamics that characterize the United States and Europe.

American literature highlights the role of religion in responding and catering to the immigrants' social needs, which can be summarized, by borrowing Hirschman's (2004) formula, by the three Rs: refuge, respectability, and resources.

Religion has been analyzed as a socially acceptable way for American immigrants to express, reformulate and convey their cultures and ethnic identities. Immigrants learn that Americans are more willing to accept religious diversity that ethnic diversity, and therefore use religion as a socially tolerated tool to build their culture and identity (Herberg, 1960; Karpathakis, 2001).

In churches and temples, immigrants "can (...) pass on their religious and cultural heritage to the following generation" (Ebaugh, Chafetz, 2000: 141). The act of going to church or a place of worship, which is often encouraged by families, seems to have a positive effect on young generations, also limiting the risk of situations of social exclusion (Zhou, Bankston, 1994). Moreover, religious organizations can increase upward mobility in second generations (Zhou, Bankston, 1998).

In the United States, religion helps transform immigrants into Americans and gives them and their children a sense of belonging. In other words, religious institutions are places where immigrants can formulate their requests to be included in the American society (Portes, Rumbaut, 2006).

\subsection{Religion as a Barrier to Integration}

In Western Europe, religion is generally perceived as an issue associated with immigrant minorities. When analyzing immigrants' religions, the focus of 
academic researchers is almost exclusively on Islam, which is regarded as a barrier or as a challenge to integration, and as a source of conflict with local customs and institutions. Some social scientists have more radical views suggesting or explicitly stating that Islam is hindering the integration of immigrant minorities and threatening the liberal values of European countries. Another topic in the social sciences literature concerns the discrimination against and restrictions on Muslims in Western Europe (religion as a vehicle for discrimination and prejudice).

Islam can become a place where to build an opposing identity: a progressive growth of a religious conscience among members of the younger generation more and more often refers to a globalized Islam, rather than to their parents' Islamic family. This process has been called re-islamization (Laurence, Vaisse, 2006). Some scholars and researchers claim that loyalty to Islam has had positive effects, such as helping young people keep away from criminality and juvenile delinquency, but there is also growing concern about the role of Islam in second-generation cultural isolationism, which may lead to acts of violence and terrorism.

An interesting perspective is the one relating to the theory of segmented assimilation (Portes, Zhou, 1993; Portes, Rumbaut, 2006): after migration, the various generations in the family pursue continuity with the culture of their home country in very different ways. Therefore, there are several possible outcomes of this:

- Consonant acculturation: it occurs when the parents and children are acculturated at the same time and speed, both in learning the culture of the host society, and in abandoning their native language and cultural traditions; in these situations there are few intergenerational conflicts;

- Dissonant acculturation: it occurs when children learn English and adopt habits and lifestyles of the host society faster than their parents do. Differences in the acculturation processes can lead to intergenerational conflicts;

- Selective acculturation: it may occur when parents and children, who have been acculturated together, preserve ties with their culture of origin. This can lead to socialization with the lifestyles of the host country without losing the most important cultural values of one's home country, in both the first and second generation.

There is another perspective in the analysis of intergenerational transmission that highlights the conflict between generations.

There is a conflict between generations when children are exposed to the new cultural inputs from the society of the country where they live and from global cultures (Caneva, 2011), while their parents want to teach them the 
values and behaviors from their homeland. Besides, sometimes parents have an embellished version of the values from their homeland, which might have changed since they left it (Foner, 2009).

Often, at the heart of this intergenerational conflict, there is some difficulty in accepting one's paternal authority (Fass, 2005), or even the discomfort and shame caused by the customs and traditions typical of one's home country (D'Alisera, 2009).

However, this conflict is not the only option in intergenerational relationships: admitting the possibility of cultural hybridization (Gomarasca, 2009) is also possible. Moro et al. (2009) remind us that the real challenge of migration is indeed that of hybridization: "...integrating into the world here, with the support of one's parents' world of origin, leads to a dynamic mix of women and men, thoughts and their evolution. Saying hybridization means saying that all forms are possible ... in an ever-changing configuration, which sometimes varies according to internal needs and in the various stages in life" (Moro, Baubet et al., 2009: 14).

By adopting their first-generation immigrant parents' point of view, children, who are surrounded by new lifestyles, can also change some cultural patterns and social customs. Adults can try and negotiate new family rules with their children, accept new norms and soften their parenting styles (Zhou, 2009). This approach shows a sort of reciprocity between generations.

The big picture thus appears to be quite articulate and complex, and it is precisely within this marked differentiation, which sometimes can be extremely ambivalent, that the transmission of values takes place in families experiencing migration.

\subsection{The Dynamics within Immigrant Families}

If, on the one hand, emigration is always an event involving the whole family, and in which the whole family changes (Gozzoli, Regalia, 2005; Valtolina, Colombo, 2012), different generations will implement different strategies to preserve-while-changing.

In Vatz-Laaroussi's study (2001) -whose suggestive title is Le familial au cour de l'immigration-, family is once again at the center both in migration trajectories and in integration dynamics. In her book, the author duly relates that not only migration, but also social participation and exercising one's citizenship are a family affair.

Sociologists studying the transmission of values claim that, in various fields such as religion, politics, or relationships between the sexes, family is what affects the most young people's socialization, whereas parents' educational methods -be they authoritarian or permissive, autocratic or democratic- only have a 
mild influence on the level of transmission of values within the family (Percheron, 1991). Coherence, persistence, visibility and the social acceptability of the message appear crucial; these are the essential dimensions for the transmission between generations, and family is the privileged place for transmission. For a long time, immigrant families have been described as a place of cultural contradictions, in which the parents' coherence and persistence is opposed to the social invisibility and unacceptability of the supported values. Observing real-life experiences in both Quebec and France, a dynamic transmission may be identified in migration trajectories. Although it does not transmit a fixed set of values and customs from one generation to another, this transmission transforms "containers, contents, transmitters and receivers" (Percheron, 1991: 94).

Migration favors a two-way (bidirectional) transmission between children and parents. Everybody must adapt to and increase knowledge and expertise. And therefore, the transmission of values involved in immigration is never linear, but is more often multi-faceted and can be understood by analyzing it at different levels.

\subsection{Semantic Transmissions}

Their aim is to transfer a meaning and an orientation within the family (such as, for instance, the importance of helping each other out). The vast majority of immigrant families describe this as a spiritual value, connected with religion or a form of cultural humanism, which is quite distant from the idea of obligation that we tend to associate it with.

\subsection{Hybrid Transmissions}

These transmissions are the strongest indicator of the family's tendency to change.

It can be noted that, in hybrid transmissions, parents', as well as children's, customs are less and less formal and automatic, and more and more contextualized and dynamic, justified on a spiritual or social level. Both the effort and the progressive re-orientation of younger generations range from the need to conform to one's family to the ability to make personal decisions (e.g. Ramadan for Muslims), without the support of the surrounding social context.

Some relevant strategies seem quite significant: for example, Catholics from Latin America join specific religious groups (Adventists or Seventh-Day Christians) as there they find an environment that looks more appropriate to the transmission of their faith and beliefs.

\subsection{Transmissions by Keywords}

The semantic meaning given to values that can have multiple meanings deserves special attention. A key concept becomes the center of, and a catalyst to, 
family transmission: it is often polysemous and multi-dimensional, and allows for several interpretations and multiple updates depending on the family members who use it -young or adults, men or women-, and on the context. An example of this is the value of respect. It is one of the key concepts of immigrant families' transmission in both Quebec and France, precisely because of its polysemy. Through nuances and contextualization, respect allows each family member to find their place within the family, and to interact with other generations, sexes, as well as society and institutions.

The transformation of the contents of the transmission often leads to switch from values to skills, due to the need to survive in an unfamiliar setting (e.g. the value of patience of Arab Muslim women in the relationships with their husbands can, in turn, become a skill for their husbands in their social relations with the Public Administration, in the labor market, or in neighborhoods).

In another survey (Helly et al., 2001) conducted by the same research team, the focus is on the transformation process of values and customs by Moroccan and Salvadoran immigrants who, in the 199os, faced different stabilization conditions than people from previous migration flows. Four are the reasons why such processes are observed within families: the importance of families in socialization processes as well as in processes of production and reproduction of models and customs; their intermediate position among the macro- and micro-sociological (individual) levels of analysis; their key role in emigration as a meeting and stabilization place for homeland cultures; and the promotion of family solidarity for most immigrants.

The research focuses on the project of transmission of values and customs and allows to identify three types of pivot (i.e. element that eases the transfer) in transmission:1) grandparents, who are the symbolic and actual actors of this process; 2) the home country, which is sometimes idealized and unwelcoming, and towards which parents develop an ambiguous relationship, which they communicate to their children to justify their emigration and offer them a potential identity; 3) networks, mainly informal ones, including members from one's home country community and from the immigrant community, which allow to recreate value-based social relations and to speak one's native language.

In a more recent article, Vatz-Laaroussi (2007) emphasizes how important family (us as a family) and social networks are. This us as a family -carrying a strong migration project, acting as a vehicle for integration into the new social life, and mediating participation in new social institutions- is also a catalyst to resilience and, at times, almost the only element of continuity in disruption and change trajectories for those who emigrate. The featured analysis follows the three dimensions that run through and make up the dynamics within immigrant families: the processes of intergenerational transmission, family 
memories and history, and resilience. This study is based on a number of surveys conducted on foreign families with children in Quebec. Its results show that neither the home country nor the parents' level of education were the single determining factors affecting the processes of intergenerational transmission or children's resilience. However, these factors lead to a range of modes of transmission of the family's history, and of children's school performances. The author's analysis allows to redefine the axes that run through family issues and immigration: family transmission is here associated with the creation of new reference points and meanings, family memories refer to a contextualized construction of the family's history within immigration, and resilience allows for each individual's re-integration into transnational networks crossing space and time boundaries. If we observe intergenerational relationships from these complicated processes, they appear as a turning point in the family's and society's transformation, which establishes a continuity that is essential to resilience, social integration and the creation of a new Self, within a different and multiple $U s$, and possibly also to the birth of a new reality.

While immigration has altered the religious structures in French society, the opposite process, i.e. the transformation of immigrants' religiousness in their new living environment (Simon, Tiberj, 2014), can also be observed. In this context, how does immigrants' religiousness evolve and differ from that of the mainstream population? How does intrafamilial religious transmission occur? And to what extent does being raised in a family where religion was important determine people's current religiousness? Is the religious sentiment more often maintained in religious minorities, particularly in the context of migration? The authors of the recent Survey on the Diversity of the French Population (Beauchemin et al., 2016) have tried to answer these questions through data analysis.

Before being a matter of personal choice, religion is transmitted by parents through socialization or, more in general, religious belonging: in other words, children are regarded as the heirs to their parents' religion. The survey reveals which religions the parents belong to, and emphasizes the importance of religion in the education people receive. Being raised in a family where religion is very important influences the formation of religious feelings: transmission happens in $85 \%$ of cases, even though with a marked decrease in the intensity of religiousness. Conversely, almost everyone who was raised in an agnostic or atheistic family claims to be equally agnostic or atheist, and less than $7 \%$ reports some degree of religiousness. Religious-mixed families, i.e. where one of the two parents has no religion, represent $10 \%$ of cases and are even rarer among immigrant families. The decrease in religion intensity from one generation to the following one is clearly expressed by this comparison: $24 \%$ of 
people between 18 and $5^{0}$ living in metropolitan France were raised in a selfdeclared religion-less family, and today $44 \%$ of people claim to have no religion.

This disaffiliation trend varies according to the religion that the parents practice. Religious disaffiliation is apparently more frequent for people raised in a Buddhist (30\% of disaffiliation), Christian and Jewish (26\%) family than for those who have Muslim parents (11\%). Intergenerational transmission can be divided into three categories:

- Secularization: religious disaffiliation from the religion of one's parents, or lower level of religiousness compared to that of one's family;

- Reproduction: same level of religiousness, including absence of religion;

- Reinforcement: higher level of religiousness than that of one's parents (including agnostics).

According to this categorization, slightly more than a quarter of people between 18 and 50 living in metropolitan France followed a secularization trajectory and consider themselves less attached to religion than their parents, two thirds maintain continuity with their families' religious feelings, and only $7 \%$ consider themselves more religious than the previous generation.

\subsection{The Relationship between Parents and Children in Transmission}

Observing the dynamics of intergenerational transmission of values in immigrant families from the mother-child relationship perspective is the aim of the research conducted in Italy by Caneva and Pozzi (2014). Through the design of qualitative interviews with immigrant mothers and children, the research analyses how mothers and children negotiate styles of transmission of values, as well as the importance of language and religion. ${ }^{1}$ The survey provides different results for Muslim and Christian women. Muslim women perceive religion as a key factor in personal identity, whereas Eastern-European Christian women perceive religious belonging as a private matter and generally attach little importance to participation in religious celebrations and Holy Scripture reading. For Muslim women, though, the little chance they have to take part in religious celebrations and their long stay in Italy lead them to distance themselves from religious practices, and this impacts their children's socialization. This distancing from rules is especially present in the younger generations and determines a sense of discomfort and shame in mothers, in so far as they have

1 The interviewees were 23 children between 14 and 20 who came to Italy to be joined with their parents again (10 Pakistanis, 9 Romanians, 2 Ukrainians, and 2 Moldovans), and 58 women (26 Bangladeshi, 7 Pakistanis, 21 Romanians, 3 Ukrainians, and 1 Moldovan) who had emigrated before, or had arrived in Lombardy through processes of family reunification. 
not been able to maintain interest in a religion. Children feign interest only when their parents are present.

For Eastern-European immigrant women, the importance of religion does not lie in regular attendance, but in its supporting and guiding role. This allows children to feel independent and autonomous, and mothers to not feel a sense of shame or defeat (the transmission of religion is regarded as a transmission of values rather than of customs).

For children, knowing and speaking their parents' native language is essential to preserve their connection with their origins and define their identity. Language is a symbolic boundary through which children define themselves and differentiate themselves from others. As for the transmission of religion, some of the young interviewees state that they go to church or the mosque and perform religious rituals (e.g. they pray). In particular, Muslim children state that religion is part of everyday life in their families. For some, religion is a way of life, a set of rules that are useful in everyday life to provide a guide to solve problems. Children feel that faith and religious practices are part of their identity and want to transfer them to their children in the future.

Despite some differences with their mothers, conflicts do not occur over religion and religious practices. This behavior is common to a lot of young people who claim to be religious, even though they go less often to church or the mosque and pray less than their parents. The decrease in religious practice is partly due to the duration of their stay in Italy. Young people who are born in Italy or who came when they were still babies are less devoted to beliefs and religious practices, whereas those who have been socialized in their homelands and have arrived in Italy in their teenage years are more devoted.

Children with Easter-European origins state that their families are not religious, and that religion is not a part of the education they receive from their parents. For these children, the transmission of values is related to other cultural issues (like, for instance, the importance of showing adults respect, or the importance of the nuclear family), which are not closely related to religion.

In conclusion, Asian Muslim families show a stronger attachment to religion, whereas Easter-European families seem less interested in religious aspects.

For Muslim women and their children, religion is still a fundamental issue. Both parents and children see the transmission of religion as a way to preserve values and moral integrity, even though second-generation children attach less importance to customs, thus creating a conflict with their parents.

However, this sense of belonging to a religious community clashes with a lower attendance at the mosque. This could be a consequence of an absence or shortage of places of worship, but also of a change in habits in a context of 
migration, or a strategy to avoid discrimination. For Eastern-European women and younger generations, religion is not closely related to identity issues and attachment to origins issues: it can be seen at most as a moral guide.

Also a recent study conducted in the Archdiocese of Milan on two generations of Muslims (Bramanti, Meda, 2016) highlights a different degree of adherence to Islam related to gender and generation. As for gender, women get predominantly higher scores in the adherence to Islam index (Closeness-Distance between self and Islam (Graphical Display); Attendance at religious practices; Orientations-Values) ( $49 \%$ high modality versus $42.3 \%$ for men), whereas, in terms of difference between generations, older people show higher levels of identification with Islam. Those who were born in Italy (second generation) actually appear in definitely lower numbers within the higher level of adherence to Islam (about $17 \%$ versus $49 \%$ of those who were born abroad or are first-generation), almost as if a secularization process is already underway for second generations.

In terms of willingness to get to know the host culture, the level of adherence to Islam seems to be neither a hindering nor an enhancing factor: both those who appear to be relatively more distant from a strong identification with religious values and those who are conversely totally devoted are actually willing to dialogue, whereas bridging social capital availability proves crucial.

\subsection{The Importance of One's Native Language}

Language, particularly one's mother's language (Medvedeva, 2012), is definitely a unique tool to transfer values within families. Language is closely related to the chance to express religious contents and preserve a sense of belonging to one's original community, especially for Muslims. Using one's native language, effectively combined with the new language, is crucial for a successful integration process (Esser, 2006; Jiménez, 2011; Ambrosini et al., 2013). However, parents and children show different abilities in learning the language of the host country: children tend to learn it faster than their parents. And this can lead children to become their parents' parents: the result is a dissonant acculturation and, eventually, a weaker parental authority, or even conflict (Choi et al., 2008).

\subsection{Generational Differences}

The author (Saint-Blancat, 2004) analyses the cultural and religious socialization of young Muslims who were born or socialized in Europe, through the generation category. The family's religious socialization, the pluralism of Islam re-affiliation paths followed by young Muslims and their current attempts at re-orientation towards faith are then examined. Lastly, identifying changes in 
the transmission process (contents and practices) highlights the queries new generations have: claiming a sort of gender identity for women through the prism of religion, a new need of citizenship, and the issue of "religious" authority. All these progresses help Islam emerge and drift away from a social construction of exception.

\subsection{Gender Differences}

This paper (Flor, Knapp, 2001) is interesting for the interpretive categories used and the final discussion highlighting the paradoxical effects related to the children's gender. ${ }^{2}$ The first introductory part proposes an open discussion about the unidirectional model (parents to children) versus a dialectical model (transactional). In these models, both parents and children are perceived as active agents in the internalization process.

Some of the parents' transmission methods are unidirectional, such as, for instance, the parental desire for the child to be religious, but there are also more dialogical methods.

In this research, the child's religiousness is regarded as a dependent variable of the parents' religiousness. In short, parents appear to have a direct influence over children, but transactional elements can also be noted. There are some interesting paradoxical effects about gender. Despite the occurrence of dialogical discussions about faith between fathers and daughters, the latter see religion as decreasingly important; a similar paradoxical, although less marked, effect was evidenced in the mother-son dyads.

\subsection{Memories}

According to Amadini (2012), in reprocessing one's obligations, heritage and sense of belonging, new generations can discover that there is a system of models, rules and values that convey the reassuring feeling of not having to start from scratch. This takes us back to memories, a topic included in the broader topic of transmission.

Among the records of the past, Assman distinguishes cultural memory from communicative memory, which includes memories from the recent past that human beings share with their peers: a typical case is generational memory. Assman underlines that this memory is built on the interaction in everyday life which is transmitted between generations by witnesses, and is less institutionalized than cultural memory, which has fixed objectifications (Assman, 2002). In religious transmission, everyday life within the family and living in the

2 Participants in this study were a subgroup of ${ }_{171}$ Caucasians living in families with two parents and one pre-adolescent child ( 84 women, 87 men). 
family's house help build an incorporated memory that can be described as communicative memory. Leccardi (2009) argues that, in family relationships, experience is incorporated as a natural behavior, which generations are a living memory of, a long memory that is present in families below the threshold of awareness.

According to Pace, the act of transmission indicates a constant movement in time: in the transfer from one generation to the other, not only do external conditions vary, but also different and somehow opposite ways of understanding and interpreting the contents of the beliefs being transmitted alternate (Pace, 2008).

This structuring of beliefs has a social, cultural and political nature. It can be interpreted as a search for new meanings of everyday life in spite of diversity as a continuous negotiation process to extend the symbolic boundaries of faith, a constant symbolic (and generational) renovation to adapt to the surrounding environment.

According to Campiche (2010), religious transmission shows a personal history building up through a relationship between the evolution of personal freedom, management of one's heritage and integration into a specific sociohistorical context.

Barrera Rivera (2001) argues that transmission is a religious language dynamics in the making, and describes it as being inseparable from the concept of group-approved memory, and here a connection between transmission and exercise of religious authority is inevitably established. According to the Brazilian author, the aim of transmission is ensuring socialization to individuals and groups within this institutional framework, by also integrating, in a balanced way, community, ethical, emotional end cultural records of identification with the religious traditions. Thus, transmission continues to be a process on which religious identities, which are temporary and partial, are built and rebuilt, based on individual experiences.

\subsection{The Role of Elderly People}

Elderly people are the leading actors in the exercise of memory. Aggoun (2003) described their (the grandparents') place in Algeria and France. Immigrant grandparents can combine their individual memory of the past -which includes a collective identity- with the present and future of the host society. They build their identity through a selective memory (a collage of elements from the homeland and host societies in a dialectical and non-dichotomous way) and by trying to find consistency and plausibility in their present and past. In doing so, the sense of belonging to their community, as an element in their personal identity, is lost in their transition from one society to the other. 
However, religion is still an element that ensures a connection between the living and the dead.

This analysis allows to explore the continuity and discontinuity of roles and functions and the transmission of memory in migration situations.

As for references to the past, the concept of generation of post-memory refers to the transmission of family memories across generations, and specifically to second generations' ties with their religious past in "an (...) oscillation between continuity and rupture" (Hirsch, 2008: 106). This is a particularly interesting concept for the generations of Jewish families' children who have not experienced the Holocaust; of Armenian descendants who have not experienced the genocide; of Catholic younger people (e.g. Focolare Movement) who have neither experienced World War II, nor taken part in the 1970s youth revolution, nor known the pre-conciliar Church or the birth of ecclesial movements.

Hervieu-Léger (2003) argues that what matters the most is not continuity itself, but the fact that continuity is the visible expression of a filiation, which is explicitly claimed by the single or collective believers and makes them members of a spiritual community including past, present and future believers.

Studies on the transmission of religious values among people who have suffered religious persecutions in their home country are missing from this analytical presentation of the main studies on the transmission of religious values in immigrant families.

This absence highlights a significant gap and also a sort of collective removal.

The findings that we have presented are obviously of enormous value and importance. This is what guided us in designing the study of persecuted groups, as the following chapters in this part will illustrate. However, the almost total lack of this specific focus highlights that, in the Western scientific community, attention has only recently been turned to this issue, which has not been thoroughly examined yet.

For immigrants, the religious persecutions that they suffered in their homeland and that have forced them to emigrate are, of course, an element of severe distress, and shed a sad light on their homeland, even though the sense of belonging to it is a major factor in the immigrants' identity. Therefore, as the interviews with the members of the Coptic community emphasize (see Chapters 19 and 20), those who have suffered discrimination or violence in their home country struggle to talk about that. 
The religious freedom that immigrants experience in host countries is thus a key factor in supporting integration and re-socialization paths for both adults and youth, provided that it does not imply that religion is a totally irrelevant fact. On the one hand, removing all the pain suffered because of religion can make the role that religion has in Western countries incomprehensible to immigrants; on the other hand, it can reduce immigrants to a sort of forced silence in order to live up to social expectations.

To this effect, the integrated model of transmission of values gains a special heuristic significance: it is a common thread running through several studies, and it combines the vertical axis (generations), horizontal axis (the group of peers) and diagonal axis of social transmission (Maliepaard, Lubbers, 2013). In particular, vertical transmission refers to the role that parents play in explicit socialization, cultural education, norms and values, and in the everyday practice of behaviors that children interiorize by observing and imitating their parents' actions. Horizontal transmission is fundamental during adolescence, when friends and peers have a growing influence on them. The diagonal axis refers to extra-familial socialization: individuals are also influenced by the social context in which they grow up, notably school (Berry et al., 1992), working environments and associations or non-profit organizations.

The present chapter hypothesizes that religious values and beliefs are a valid support in integration and cohesion processes for migrant families. The framework within which this hypothesis can be verified refers to the four key concepts presented in Chapter 2.

Based on the empirical evidence provided, it can be stated that the families' commitment in the transmission of religious values is definitely aimed at building an individual and family identity in the contexts of origin. It can also be stated that this effort tends to persist at least in first-generation immigrants even in the host country.

If it is true that religious values are mainly conveyed within family relationships, it is also true, as research has found, that they are challenged by the social background of the host country, which is new to immigrants.

In European host countries and in Italy in particular, migrants experience freedom of religion, as a formal right, which, however, plays a barely active role in defining a new right to citizenship and making places of public life accessible.

This condition poses a threat: leaving families alone in their efforts to pass down values. However, on the other hand, it adds value to ethnic communities, as well as to local Christian Churches and Parishes, by investing them with a supporting role to families in this task.

Unfortunately, in Italy, there is still no adequate empiric research about these dynamics, due to the complexity of the investigation (as the first findings presented in the chapters about the Coptic families and their children who 
were targeted in this study will illustrate), and also to a sort of removal of the religious issue as a cause of migration.

This silence is dangerous and has to be purposefully tackled and studied, in order to prevent the courage and self-sacrifice of the many people who have risked or given up their lives from crashing into a wall of silence and denial. 